\title{
Prevalence and recurrence of bacteraemia in hospitalised people who inject drugs - a single Centre retrospective cohort study in Denmark
}

Sara Thønnings ${ }^{1}$, Filip Jansåker ${ }^{2,3^{*}}$ (D), Christoffer Sundqvist ${ }^{4}$, Rebekka Faber Thudium ${ }^{5}$, Susanne Dam Nielsen ${ }^{5,6}$ and Jenny Dahl Knudsen ${ }^{2,4}$

\begin{abstract}
Background: People who inject drugs (PWID) have increased risk of acquiring blood-transmitted chronic viral infections such as Hepatitis B (HBV), Hepatitis C (HCV) and Human Immunodeficiency Virus (HIV) as well as increased risk of acquiring bacterial infections. We aimed to identify and describe bacteraemic episodes, their recurrence rates, predictive and prognostic factors amongst hospitalised PWID.

Methods: In this retrospective cohort study, we included 257 hospitalised PWID during 2000-2006 with follow up at the Department of Infectious Diseases, Hvidovre Hospital, Denmark. Data collection included comorbidity (HBV-, HCV-, HIV-, and psychiatric comorbidities), social information (contact to an addiction treatment centre, homelessness), opioid substitution treatment (OST), treatment completion and microbiology findings. There was a 10-years follow-up regarding mortality.

Results: The study identified 257 patients classified as PWID. Of these, 58 (22.6\%) had at least one episode of bacteraemia during their first hospital admission. Recurrence was found in 29 (50.0\%) of the bacteraemia cases. Staphylococcus aureus was the dominant microorganism of both first and recurrent episodes with 24 (41.4\%) and nine (31.4\%) of cases, respectively. A psychiatric diagnose was significantly associated with a lower risk of bacteraemia in the multivariate analysis (OR: $0.29,[95 \% \mathrm{Cl}: 0.11-0.77], P=0.01)$. Mortality was significantly higher in patients with bacteraemia (17.2\% vs. 3.0\%, $P<0.01$, OR: 6.67 [95\%Cl: $2.33-20], P<0.01$ ).

Conclusions: In hospitalised PWID, bacteraemia was found in $22.6 \%$ and was associated with at higher mortality. The most common microorganism of bacteraemia was S. aureus. Psychiatric comorbidity was significantly associated with a lower risk of bacteraemia.
\end{abstract}

Keywords: PWID, Bacteraemia, Recurrence, Mortality, S. aureus

\footnotetext{
* Correspondence: Erik.Filip.Jansaaker@regionh.dk

${ }^{2}$ Department of Clinical Microbiology, Copenhagen University Hospital, Rigshospitalet, Copenhagen, Denmark

${ }^{3}$ Center for Primary Health Care Research, Lund University, Malmö, Sweden

Full list of author information is available at the end of the article
} 


\section{Background}

Denmark has a population of approximately 5.7 million residents, and it is estimated that 33,074 people (5.8/ 1000 inhabitants) have persistent usage of illicit drugs. About 13,000 (2.3/1000 inhabitants) of these are people who inject drugs (PWID) [1].

Mortality among PWID is known to be much higher than in the general population, and bacterial infections are common causes for hospitalisations and death [2]. As a result of intravenous (IV) drug administration, most bacterial infections are caused by the subjects' own commensal flora, with Staphylococcus aureus being the most common cause of bacteraemia as well as skin- and softtissue infections [3, 4]. Data regarding bacteraemia in PWID in Denmark is sparse, yet previous studies indicate a higher prevalence of bacteraemia in this patient category [5, 6]. Bacteraemia is a serious condition that strongly affects the patients morbidity and mortality, and bacteraemic episodes and their recurrence rates among the general population have previously been investigated in Denmark showing a recurrence rate of $12 \%$ within one year [7]. Compliance to treatment is a challenge for PWID, which may influence the recurrence rates of bacteraemia in this population. In addition, PWID have a high prevalence of chronic viral infections [8]. Hence, the aims of this study were to describe bacteraemic episodes and their recurrence rates in PWID admitted to the hospital over a six-year period. Moreover, we wanted to determine prognostic factors for bacteraemic episodes and their recurrence and study associations with mortality.

\section{Methods}

\section{Setting and data sources}

This retrospective cohort study included patients from 2000 to 2006 admitted at Copenhagen University Hospital, Hvidovre, Denmark. All Danish residents are given a unique 10-digit identification number used for all health care contacts [9]. Health care in Denmark is taxsupported and free of charge for all Danish residents, including substance abuse treatment [1].

Patients were identified retrospectively using a database search of hospital admissions to Copenhagen University Hospital, Hvidovre from 1st January 2000 to 31st December 2006. The following search-terms were used: dependence, chronic HCV infection, endocarditis, abscesses and osteomyelitis. Patients identified during the primary search were further screened through medical records. Inclusion criteria were age more than 18, hospital admission and drug abuse including injection of drugs. No exclusion criteria were applied. Further data regarding the patients included in the retrospective cohort were collected from medical records using a standardised data collection form. Data collected at the time of first hospital admission were: Contact to an addiction treatment center, homelessness, co-morbidities (i.e. HBV-, HCV-, HIV-, psychiatric comorbidities and use of psychiatric treatments), opioid substitution treatment (OST), and treatment completion. Furthermore, the dataset included microbiological findings between 1st January 2000 and 31st July 2008 which were retrieved from the local microbiology database. The cohort had a 10-year follow up regarding mortality. Supplementary Fig. 1 present the timeline of the study.

\section{Microbiology}

A clinical physician did all microbiological samplings at clinical indications. Blood cultures were performed using two sets of one aerobic and one anaerobic bottle (BactAlert, bioMerieux, France). The positive blood cultures were sub-cultured, and the microorganisms were identified by standard methods [10], and susceptibility testing was performed by disc diffusion methods according to guidelines provided by EUCAST (www.eucast.org).

\section{Viral infections and bacteraemia}

A clinical physician ordered all laboratory analyses. HBsAg, anti-HBs, anti-HCV and anti-HIV were analysed using Electrochemi-luminescens Immunoassay "ECLIA" (COBAS6000, Roche Diagnostics GmbH, Mannheim, Germany). HCV-RNA were analysed using real-time transcription-mediated amplification (Panther system, Hologic Inc., San Diego, CA).

HBV-status was classified according to HBsAg and anti-HBs. The presence of HBsAg was defined as acute or chronic infection and presence of anti-HBs was defined as resolved infection or due to immunization. $\mathrm{HCV}$-status was classified according to detectable HCVRNA and anti-HCV; Presence of only HCV-RNA was defined as an acute infection, presence of both HCVRNA and anti-HCV as chronic infection and presence of only anti-HCV as resolved infection. HIV-status was recorded based on anti-HIV. A bacteraemic episode was defined as growth of any blood culture isolate within 48 $\mathrm{h}$ from admission to the hospital. Recurrent bacteraemia was defined as growth of any other blood culture isolate beyond $48 \mathrm{~h}$ from the first isolate from a new blood culture set, or the same microorganism more than 30 days after the initial bacteraemia within two years. Coagulasenegative staphylococci, Corynebacterium species and Cutibacterium acnes were classified as contaminants, unless they were isolated from two or more separate blood-culture sets. Fungaemia was for convenience also classified as bacteraemia.

\section{Medication, comorbidity and mortality}

Any prescribed dose of methadone or buprenorphin at the time of admission was defined as OST. Psychiatric 
comorbidity was defined as any psychiatric diagnosis prior to hospital admission. Patients were registered as homeless when they had no residential address and were not residents on an institution. Contact to an addiction treatment center was defined as any present contact to an addiction treatment centre. Treatment was registered as completed if the patient followed the scheduled treatment plan (e.g. was not discharged prior to schedule treatment ending or failed to show in the outpatient clinic).

The mortality was defined as all-cause mortality from the day of hospital admission. In case of recurrent bacteraemia the latest admission with a positive blood culture was used to calculate all-cause mortality. Mortality was either 30-day mortality or 10-year mortality.

\section{Statistical analysis}

Continuous data were presented as medians with interquartile ranges. Categorical data were analysed with the Fisher's exact test with odds ratio (OR) presented with 95\% confidence interval (95\% CI). Univariate OR with 95\% CI for bacteraemia and recurrence of bacteraemia were calculated using logistic regression. Variables with $P<0.30$ were included in multivariate analyses for predictive factors of bacteraemia. Survival curves were presented as Kaplan-Meier curves and compared using the Log-rank test. No patients were excluded in the analysis. Two-sided significance was tested with the assumption of $P<0.05$ as statistically significant.

The Statistical Package for Social Sciences (version 26.0; SPSS, IBM) was used for the analysis.

\section{Ethical considerations and data protection approval}

The Danish Data Protection Agency approved the study (7-505-29-369/1).

\section{Results}

\section{Baseline data}

Figure 1 shows a flow chart of the included patients. In the six-year study period from 2000 to 2006, we identified 257 hospitalised PWID. Acute or chronic HBVinfection were found in $4.3 \%$ of the patients and 19.5\% had a resolved infection or were immunised (Table 1). Chronic hepatitis C was seen in $39.7 \%$ of all patients, and another $22.6 \%$ could not be distinguished from either chronic or resolved infection because of a missing data on HCV-RNA. Almost half of the patients were HIV-positive. Table 1 and Supplementary Table 1 shows the full patients status regarding, hepatitis $\mathrm{B}$, hepatitis $\mathrm{C}$ and HIV.

In the cohort, 58 (22.6\%) PWID had at least one episode of bacteraemia. Blood cultures from the remaining 199 patients was either negative or blood cultures were

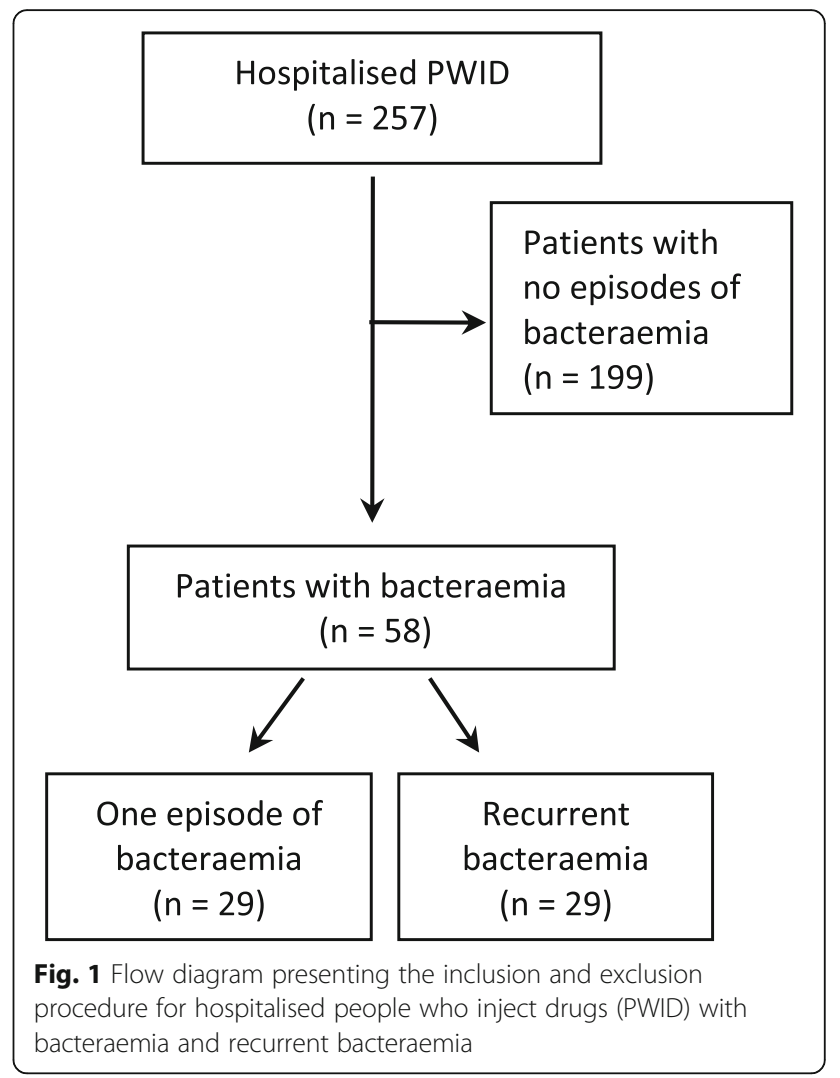

not performed. Recurrent bacteraemia was found in 29 patients, of whom 27 had recurrence within one year.

\section{Microbiological findings in first and recurrent bacteraemia}

The first episode of bacteraemia was in 48 patients mono-microbial (82.8\%). Mono-microbial Gram-positive bacteraemia accounted for $43(74.1 \%)$ of all the first episodes $(n=58)$ and $22(75.9 \%)$ of the recurrent episodes $(n=29)$.

Staphylococcus aureus accounted for 24 (41.4\%) of all first episodes of bacteraemia and nine (31.0\%) of recurrent episodes. Streptococcus pneumoniae was the second most common microorganism with six (10.3\%) of all first episodes. Only one bacteraemic episode was caused by Escherichia coli.

Among the recurrent episodes, non-haemolytic streptococci were the second most common finding with three $(10.3 \%)$ cases. Two episodes with yeasts were both in recurrent episodes. Of all the recurrent episodes, recurrence of the presumed same microorganism as in the first bacteraemic episode was only found for S. aureus in six out of ten episodes (see Supplementary Table 2).

\section{Predictors of bacteraemia and recurrence}

Patients with bacteraemia $(n=58)$ were compared to patients without bacteraemia $(n=199)$ (Table 2). In 
Table 1 Clinical characteristics of 257 hospitalised people who inject drugs

\begin{tabular}{lll}
\hline Clinical characteristics: & PWID $n=257(\%)$ \\
\hline Age (median) (IQR) & & $38(34-45)$ \\
HBV-status & Acute/Chronic & $11(4.3)$ \\
& Resolved/Immunised & $50(19.5)$ \\
& Negative & $79(30.7)$ \\
& Unknown & $117(45.5)$ \\
HCV-status & Acute & $1(0.4)$ \\
& Chronic & $102(39.7)$ \\
& Resolved & $12(4.7)$ \\
Chronic or resolved & $58(22.6)$ \\
HIV-status & Negative & $8(3.1)$ \\
& Unknown & $76(29.6)$ \\
HIV and HCV co-infection & $127(49.4)$ \\
Psychiatric comorbidity & $101(39.3)$ \\
Opioid substitution treatment & $29(11.3)$ \\
Homeless & Negative & $88(34.2)$ \\
Contact to an addiction treatment center & $53(20.6)$ \\
\hline
\end{tabular}

If not stated otherwise, results are presented as $\mathrm{n}(\%)$ Abbreviations: PWID people who inject drugs, HIV Human Immunodeficiency Virus, $H B V$ hepatitis $B, H C V$ hepatitis

univariate analysis psychiatric comorbidities were less common among patients with bacteraemia (8.6\% vs. 24.1\%, OR: 0.30 [95\%CI: 0.11-0.76], $P=0.01$ ). Adjusting for age, HBV, HCV and HIV in the multivariate analyses, psychiatric comorbidities were still significantly associated with a lower risk of bacteraemia (adjusted odds ratio (aOR): 0.29 [95\%CI: 0.11-0.77], $P=0.01$ ). Comparing patients with recurrent bacteraemia $(n=29)$ to patients without recurrence $(n=29)$ no significant predictive factors were found (Table 3 ).

\section{Mortality}

The 30-day mortality in the cohort was 6.2\% (16/257). Patients with bacteraemia had a significantly higher mortality compared to non-bacteraemic patients both in univariate analysis $(17.2 \%$ vs. $3.0 \%$, OR: 6.67 [95\%CI: 2.33-20], $P<0.01$ ) and comparing the survival curves (Log-rank test $P<0.01$, Fig. 2). Mortality was still significantly higher 10 years after $(65.5 \%$ vs. $48.7 \%$, OR: 2.00 [95\%CI: 1.09-3.70], $P=0.03$ ).

Subgroup analysis of the patients with bacteraemia showed no difference in 10-years mortality when comparing patients with recurrent bacteraemia vs. nonrecurrent bacteraemia $(65.5 \%$ vs. $65.5 \%$, OR $1.00 \mathrm{CI}$ $0.34-2.95, P=1.00)$.

\section{Discussion}

In this retrospective cohort study, we found that $22.6 \%$ of hospitalised PWID had at least one episode of bacteraemia during their hospital admission. Bacteraemia was associated with significantly higher mortality, which is in accordance with previous studies [11-14]. In addition, half of the cohort with bacteraemia had recurrent bacteraemia during the study period. Studies among the general population have reported recurrence rates of 9$12 \%[7,15]$. However, higher incidence of recurrence among PWID was expected. First, a higher incidence of overall bacteraemia leads to a higher rate of recurrence due to increased risk of new bacteraemia episodes added to treatment failures. Secondly, PWID are often in a poor socio-economic position and behavioural factors play a large role in recurrence [16].

Intravenous drug use is associated with a higher incidence of different types of infections [2, 17, 18]. S. aureus was the dominant microorganism in the first

Table 2 Predictive factors of bacteraemia in hospitalised people who inject drugs

\begin{tabular}{|c|c|c|c|c|c|c|c|}
\hline \multirow[t]{2}{*}{ Predictors } & \multirow{2}{*}{$\begin{array}{l}\text { Total } n= \\
257(\%)\end{array}$} & \multirow{2}{*}{$\begin{array}{l}\text { Bacteraemia } \\
n=58(\%)\end{array}$} & \multirow{2}{*}{$\begin{array}{l}\text { Non-bacteraemia } \\
n=199(\%)\end{array}$} & \multicolumn{2}{|c|}{ Univariate analysis } & \multicolumn{2}{|c|}{ Multivariate analysis $P<0.3$} \\
\hline & & & & $\overline{\mathrm{OR}^{\mathrm{A}}\left(95 \% \mathrm{Cl}^{\mathrm{B}}\right)}$ & $P$ value & $\overline{\text { Adjusted } \mathrm{OR}^{\mathrm{A}}\left(95 \% \mathrm{Cl}^{\mathrm{B}}\right)}$ & $P$ value \\
\hline Age (years) & $39(34-45)$ & $40(34-45)$ & $39(33-44)$ & $1.02(0.99-1.05)$ & 0.27 & $3.46(1.30-9.23)$ & 0.21 \\
\hline HBV (acute or chronic) & $11(4.3)$ & $4(6.9)$ & $7(3.5)$ & $2.03(0.57-7.20)$ & 0.27 & $1.42(0.34-5.95)$ & 0.63 \\
\hline HCV (acute or chronic) & $103(40.1)$ & $17(29.3)$ & $85(42.7)$ & $0.56(0.30-1.05)$ & 0.07 & $0.57(0.30-1.08)$ & 0.08 \\
\hline HIV positive & $127(49.4)$ & $25(43.1)$ & $102(51.3)$ & $0.72(0.40-1.30)$ & 0.30 & $0.72(0.39-1.32)$ & 0.29 \\
\hline Psychiatric comorbidity & $53(20.6)$ & $5(8.6)$ & $48(24.1)$ & $0.30(0.11-0.76)$ & 0.01 & $0.29(0.11-0.77)$ & 0.01 \\
\hline Opioid substitution treatment & $210(81.7)$ & $52(89.7)$ & $158(79.4)$ & $2.25(0.90-5.60)$ & 0.84 & & \\
\hline Homeless & $74(28.8)$ & $14(24.1)$ & $60(30.2)$ & $0.74(0.38-1.45)$ & 0.41 & & \\
\hline Contact to an addiction treatment center & $169(65.8)$ & $40(69.0)$ & $129(64.8)$ & $1.21(0.64-2.26)$ & 0.64 & & \\
\hline
\end{tabular}

${ }^{\mathrm{A} O d d s}$ Ratio (OR); ${ }^{\mathrm{B}}$ Confidential Interval (CI). $P<0.05$ in bold

If not stated otherwise, results are presented as $\mathrm{n}(\%)$

Abbreviations: PWID people who inject drugs, HIV Human Immunodeficiency Virus, HBV hepatitis $B, H C V$ hepatitis $C$ 
Table 3 Predictive factors of recurrent bacteraemia in hospitalised people who inject drugs

\begin{tabular}{|c|c|c|c|c|c|}
\hline Predictors & Total $n=58(\%)$ & $\begin{array}{l}\text { Bacteraemia with } \\
\text { recurrence } n=29(\%)\end{array}$ & $\begin{array}{l}\text { Non-recurrent bacteraemia } \\
n=29(\%)\end{array}$ & $\mathrm{OR}^{\mathrm{A}}\left(95 \% \mathrm{Cl}^{\mathrm{B}}\right)$ & $P$ value \\
\hline Age (years) & $40(35-45)$ & $39(31-43)$ & $40(37-47)$ & $0.93(0.86-1.01)$ & 0.10 \\
\hline HBV (acute or chronic) & $4(6.9)$ & $1(3.4)$ & $3(10.3)$ & $0.31(0.03-3.17)$ & 0.61 \\
\hline HCV (acute or chronic) & $17(29.3)$ & $6(20.7)$ & $11(37.9)$ & $0.43(0.13-1.38)$ & 0.25 \\
\hline HIV positive & $24(41.4)$ & $12(41.4)$ & $12(41.4)$ & $0.87(0.31-2.43)$ & 1.00 \\
\hline Psychiatric comorbidity & $5(8.6)$ & $1(3.4)$ & $4(13.8)$ & $0.22(0.02-2.13)$ & 0.35 \\
\hline Opioid substitution treatment & $52(89.7)$ & 28 (96.6) & $24(82.8)$ & $5.88(0.64-50.00)$ & 0.19 \\
\hline Homeless & $14(24.1)$ & $5(17.2)$ & $9(31.0)$ & $2.16(0.62-7.49)$ & 0.36 \\
\hline Contact to an addiction treatment center & $40(69.0)$ & $21(72.4)$ & $19(65.5)$ & $1.39(0.45-4.17)$ & 0.78 \\
\hline Treatment completed & $17(29.3)$ & $11(37.9)$ & $6(20.7)$ & $2.48(0.77-8.04)$ & 0.16 \\
\hline
\end{tabular}

${ }^{\mathrm{A} O d d s}$ Ratio (OR); ${ }^{\mathrm{B} C o n f i d e n t i a l}$ Interval (Cl)

If not stated otherwise, results are presented as $\mathrm{n}(\%)$

Abbreviations: PWID people who inject drugs, HIV Human Immunodeficiency Virus, HBV hepatitis B, HCV hepatitis C

bacteraemia episodes (41.4\%) and in the recurrent episodes (31.0\%). S. aureus has been shown to be the most common microorganism among PWID [19-22]. PWID are also known to have a higher rate of nasal and skin colonisation of $S$. aureus [23]. The high prevalence of $S$. aureus found in our study is in contrast to the general Danish population, where $S$. aureus has been shown to account for $17 \%$ of all bacteraemia [7]. In our study there was only one case of $E$. coli bacteraemia - the most common pathogen in bacteraemia in the general Danish population [24]. However, this cohort consisted of relatively young patients whom injected illicit drugs through the skin where $S$. aureus is prominent [3, 4], whereas E. coli bacteraemia is more common in an elderly population and in connection with an infection focus in the urinary tract or abdomen [25]. Considering the low number of subjects in our study, we do not consider this finding to be of evidence that PWID have lower risk of $E$. coli bacteraemia than the general population. However, it indicates that $S$. aureus has a greater prevalence as causative agent in bacteraemia in this population. Simultaneously, there seems to be an increased risk of recurrence in particularly $S$. aureus bacteraemia. S. pneumoniae was the second most common

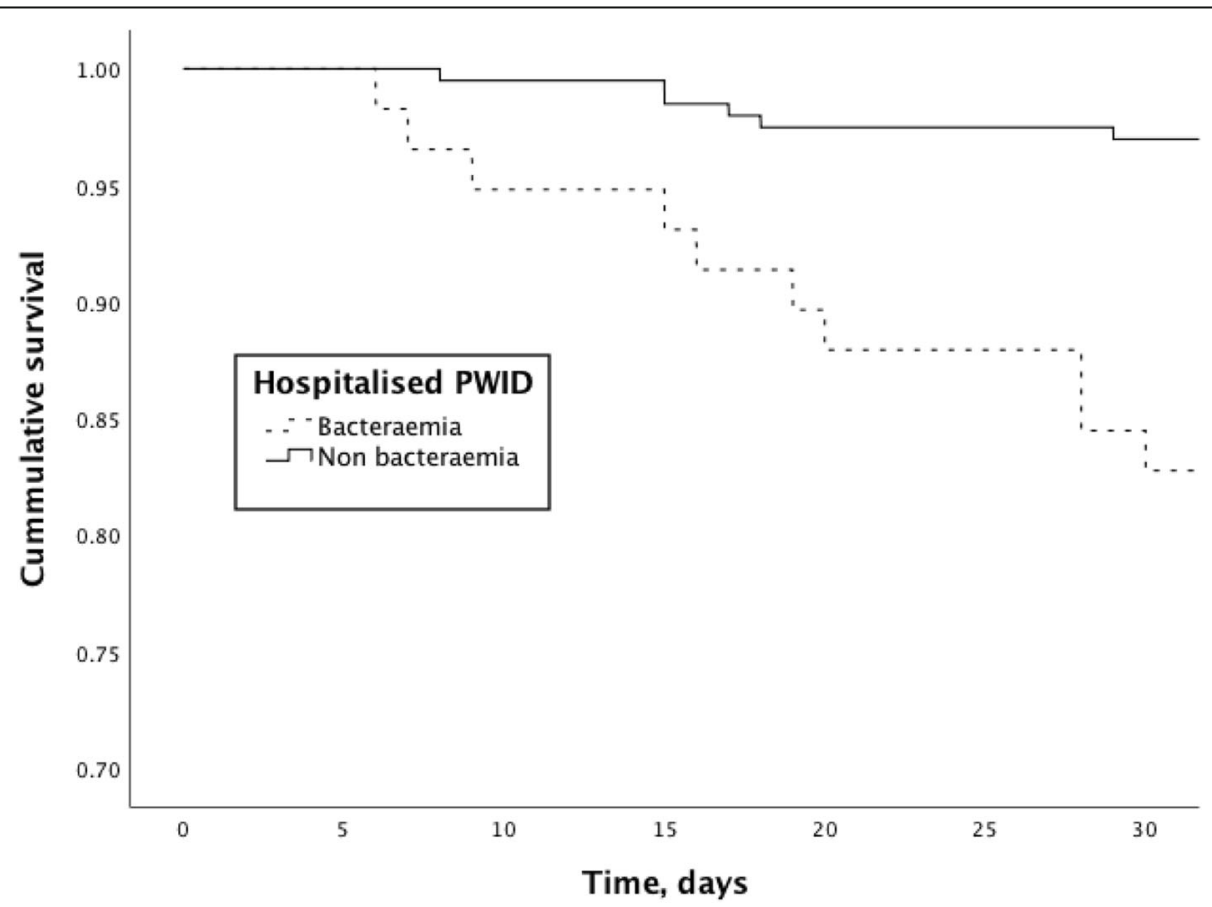

Fig. 2 Survival curves showing mortality amongst hospitalised people who inject drugs stratified on bacteraemia. Log Rank Test $P<0.01$ (Kaplan-Meier plot) 
microorganism of first bacteraemia episodes (10.3\%), which supports a previous study showing that pneumonia is one of the most common infections among PWID [4]. The cohort had a high prevalence of co-infections, yet we found no significant association between coinfections and bacteraemia.

When studying predictive factors of bacteraemia, we found that psychiatric comorbidity was significantly associated with a lower risk of bacteraemia. This group did not differ from the rest of the cohort, except with regards to a lower risk of bacteraemia (data not shown). One explanation of this finding could be, that patients with psychiatric comorbidity in connection to their psychiatric treatment are more closely monitored. Hence, treatment for e.g. cutaneous abscesses would be initiated more promptly compared to other PWID.

Our study has some important limitations. The study design was retrospective and information regarding the cohort relies on previously recorded data: Firstly, not all hospital admissions related to intravenous drug abuse may not have been included in the study, since the primary screening in hospital database was done by using a limited set of search-terms. Secondly, data regarding gender, origin of infection, antibiotic and antiviral treatment and compliance were missing.

Nevertheless, the study has advantages; firstly, the personal identification number system used in Denmark that makes follow-up very easy and accurate; secondly, long study inclusion period (6 years) resulted in a larger number of cases with a long follow up period (10 years); thirdly, comprehensive questionnaires (from hospital journal database) and complete microbiological data were found for each patient.

\section{Conclusion}

The incidence of bacteraemia and recurrent bacteraemia was high among hospitalised PWID and significantly associated with higher mortality. The most common microorganism of bacteraemia was $S$. aureus, which should be considered in empirical therapy, guided by local resistance-rates. Psychiatric comorbidity was significantly associated with a lower risk of bacteraemia, possibly due to a closer monitoring by the health care system of these patients.

\section{Supplementary information}

Supplementary information accompanies this paper at https://doi.org/10. 1186/s12879-020-05357-0.

Additional file 1: Supplementary Figure 1. Timeline for inclusion, gathering of microbiology data and follow up regarding hospitalised people who inject drugs with bacteraemia and recurrent bacteraemia.

Additional file 2: Supplementary Table 1. The HBV-, HCV- and HIVstatus for hospitalised people who inject drugs.
Additional file 3: Supplementary Table 2. Blood culture isolates detected in hospitalised people who inject drugs.

\begin{abstract}
Abbreviations
aOR: adjusted odds ratio; Cl: Confidence interval; HBV: Hepatitis B;

HCV: Hepatitis C; HIV: Human Immunodeficiency Virus; IV: Intravenous; OR: Odds ratio; OST: Opioid substitution treatment; PWID: People who inject drugs
\end{abstract}

\section{Acknowledgments}

We want to thank the personnel at the Departments of Clinical Microbiology and Infectious Diseases at Hvidovre Hospital working at the time of the study period.

\section{Authors' contributions}

FJ, ST and CS were the primary investigators of the project. Concept and design: SDN and JDK. Acquisition of data: SDN, JDK, RFT and ST. Translating raw data to electronic database: CS and RFT. Analysis of data: CS and ST. Interpretation of data: all authors. Drafting of the manuscript: FJ, ST, CS and JDK. Critical revision of the manuscript for important intellectual content: SDN, JDK, FJ, and ST. Administrative, technical, or material support: JDK, SDN, RFT and FJ. Supervision: SDN and JDK. All authors take responsibility for the integrity of the data and the accuracy analysis and interpretation of data and have approved the final version of the manuscript.

\section{Funding}

The Departments of Clinical Microbiology, Copenhagen University Hospital (Hvidovre Hospital and Rigshospitalet). The funding bodies had no deciding role in planning the design of the study and collection, analysis, and interpretation of data and in writing the manuscript. Open access funding provided by Lund University.

\section{Availability of data and materials}

The datasets generated during the current study are not publicly available due to the sensitive personal data in a small cohort but are available from the corresponding author on reasonable request.

\section{Ethics approval and consent to participate}

The Danish committee on biomedical research ethics for the capitol region of Denmark approved the study (7-505-29-369/1). Registry-based research does not require informed consent in Denmark.

\section{Consent for publication \\ Not applicable.}

\section{Competing interests}

All authors have completed the ICMJE uniform disclosure form (available on request from the corresponding author) and declare: no support from any organisation for the submitted work; no financial relationships with any organisations that might have an interest in the submitted work in the previous three years, no other relationships or activities that could appear to have influenced the submitted work.

\section{Author details}

${ }^{1}$ Department of Virus \& Microbiological Special Diagnostics, Statens Serum Institute, Copenhagen, Denmark. ${ }^{2}$ Department of Clinical Microbiology, Copenhagen University Hospital, Rigshospitalet, Copenhagen, Denmark. ${ }^{3}$ Center for Primary Health Care Research, Lund University, Malmö, Sweden. ${ }^{4}$ Department of Clinical Microbiology, Copenhagen University Hospital, Hvidovre Hospital, Hvidovre, Denmark. ${ }^{5}$ Department of Infectious Diseases, Copenhagen University Hospital, Rigshospitalet, Copenhagen, Denmark.

${ }^{6}$ Department of Infectious Diseases, Copenhagen University Hospital, Hvidovre Hospital, Hvidovre, Denmark.

Received: 11 December 2019 Accepted: 17 August 2020 Published online: 26 August 2020

\section{References \\ 1. Narkotikasituationen I Danmark 2017. Available at: www.sst.dk. (Accessed: 6th September 2019).}


2. Scheidegger C, Zimmerli W. Infectious complications in drug addicts: sevenyear review of 269 hospitalized narcotics abusers in Switzerland. Rev Infect Dis. 1989;11:486-93.

3. Lavender TW, McCarron B. Acute infections in intravenous drug users. Clin Med J Royal Coll Phys London. 2013;13:511-3.

4. Palepu A, et al. Hospital utilization and costs in a cohort of injection drug users. C Can Med Assoc J. 2001;165:415.

5. Koch $\mathrm{K}$, et al. Socioeconomic inequalities in risk of hospitalization for community-acquired bacteremia: a Danish population-based case-control study. Am J Epidemiol. 2014;179:1096-106.

6. Aagaard T, et al. Long-term mortality after Staphylococcus aureus spondylodiscitis: a Danish nationwide population-based cohort study. J Inf Secur. 2014;69:252-8.

7. Jensen US, et al. Recurrent bacteraemia: a 10-year regional populationbased study of clinical and microbiological risk factors. J Inf Secur. 2010;60: 191-9.

8. Degenhardt $\mathrm{L}$, et al. Global prevalence of injecting drug use and sociodemographic characteristics and prevalence of HIV, HBV, and HCV in people who inject drugs: a multistage systematic review. Lancet Glob Heal. 2017;5:e1192-207.

9. Schmidt M, et al. The Danish National Patient Registry: a review of content data quality, and research potential. Clin Epidemiol. 2015;7:449-90.

10. Murray, P. R. \& American Society for Microbiology. Manual of clinical microbiology. (ASM Press, 1999).

11. Martin GS, Mannino DM, Eaton S, Moss M. The epidemiology of sepsis in the United States from 1979 through 2000. N Engl J Med. 2003;348:1546-54

12. Goto M, Al-Hasan MN. Overall burden of bloodstream infection and nosocomial bloodstream infection in North America and Europe. Clin Microbiol Infect. 2013;19:501-9.

13. Laupland KB, Pasquill K, Parfitt EC, Naidu P, Steele L. Burden of communityonset bloodstream infections, Western interior, British Columbia, Canada. Epidemiol Infect. 2016;144:2440-6.

14. Nielsen SL, et al. Bacteremia is associated with excess long-term mortality: a 12-year population-based cohort study. J Inf Secur. 2015;70:111-26.

15. Capdevila JA, et al. Incidence and risk factors of recurrent episodes of bacteremia in adults. Arch Intern Med. 1994;154:411-5.

16. Wiese $L$, et al. A nationwide study of comorbidity and risk of reinfection after Staphylococcus aureus bacteraemia. J Inf Secur. 2013;67:199-205.

17. Louria DB, Hensle T, Rose J. The major medical complications of heroin addiction. Ann Intern Med. 1967;67:1-22.

18. Cherubin CE, Sapira JD. The medical complications of drug addiction and the medical assessment of the intravenous drug user: 25 years later. Ann Intern Med. 1993;119:1017-28.

19. Summanen $\mathrm{PH}$, et al. Bacteriology of skin and soft-tissue infections: comparison of infections in intravenous drug users and individuals with no history of intravenous drug use. Clin Infect Dis. 1995;20:S279-82.

20. Spijkerman IJB, Van Ameijden EJC, Mientjes GHC, Coutinho RA, Van Den Hoek A. Human immunodeficiency virus infection and other risk factors for skin abscesses and endocarditis among injection drug users. J Clin Epidemiol. 1996:49:1149-54.

21. Levine DP, Crane LR, Zervos MJ. Bacteremia in narcotic addicts at the Detroit medical center. II infectious endecarditis: A prospective comparative study. Rev Infect Dis. 1986;8:374-96.

22. Crane LR, Levine DP, Zervos MJ, Cummings G. Bacteremia in narcotic addicts at the detroit medical center. I. Microbiology, epidemiology, risk factors, and empiric therapy. Rev Infect Dis. 1986;8:364-73.

23. Bassetti S, Battegay M. Staphylococcus aureus infections in injection drug users: risk factors and prevention stategies. Infection. 2004;32:163-9.

24. Wilson J, et al. Trends among pathogens reported as causing bacteraemia in England, 2004-2008. Clin Microbiol Infect. 2011;17:451-8.

25. Laupland KB, Gregson DB, Church DL, Ross T, Pitout JDD. Incidence, risk factors and outcomes of Escherichia coli bloodstream infections in a large Canadian region. Clin Microbiol Infect. 2008;14:1041-7.

\section{Publisher's Note}

Springer Nature remains neutral with regard to jurisdictional claims in published maps and institutional affiliations.

\section{Ready to submit your research? Choose BMC and benefit from:}

- fast, convenient online submission

- thorough peer review by experienced researchers in your field

- rapid publication on acceptance

- support for research data, including large and complex data types

- gold Open Access which fosters wider collaboration and increased citations

- maximum visibility for your research: over $100 \mathrm{M}$ website views per year

At BMC, research is always in progress.

Learn more biomedcentral.com/submissions 